Der Durchmesser des Hutes betrug 3-6 Centim. Die Länge des Stieles 4-5 Ctm. Mit Ausnahme des sehr entwickelten Stieles stünde der Pilz dem Pleurotus petaloides Bull. nahe.

Ich würde vorschlagen, denselben Pleurotus sulcato-jugatus zu neunen.

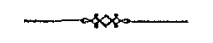

\title{
Die Rosenflora von Travnik in Bosnien.
}

\author{
Von J. B. Wiesbaur S. J. \\ (Fortsetzang.)
}

\section{Tomentosae.}

13. Rosa resinosa Sternb. Jedenfalls in einer sehr nahe stehenden Form "versus $\boldsymbol{R}$. resinosam. Sicher aus der Gruppe der $\boldsymbol{R}$. mollis (Sm.) Crépin $=R$. mollissima Borb., was die drüsig gewimperten Petalen und auch die verticillen Stacheln beweison. Aber die Blättchen sind dünner, mehr grün, an der Basis abgerundet bis herzförmig $=R$. resinosa Sternb. und von dieser nur in den verticillen dimorphen Stacheln und den einzelnen Blüthen abweichend. Von der forma umbratica Borb. unterscheidet sie sich durch die kleineren, mehr grauen Blättchen und die reichere Serratur. Allem Anscheine nach ist sie eine locale, zur Rosa Arduennensis Crép. sich hinneigende Modification, wie solche (jedoch weniger drüsig und behaart) auch um Gastein vorkommt. Vergl. Crépin VI. p. 769 (extr. p. 109)“. Keller.

Am Vlašic an der Grenze der Holzregion (leg. Brandis). Nach schriftlicher Mittheilung des Entdeckers sebr scharf riechend, etwa wie das bekannte Bleistiftholz oder wie Sandelholz.

14. Rosa tomentosa Sm.

a. R. subglobosa Sm. Am Südabhange des Velenica-Gebirges gegen Kasapovic (1. Brandis). - Eine abweichende Form ist "f. calostephana. Syn. $\boldsymbol{R}$. calostephana Gdgr. tab. 3916, R. eriophlaea Gdgr. tab. 3925, $R$. albescens Gdgr. tab. 3905, $R$. tomentosa f. scabriuscula Christ (non Baker) p. p. max." (Keller.) So im Thalgrund südlich vom Militär-Barakenspital bei Travnik (leg. Brandis).

B. R. Seringeana Godr. f. umbrigena (Keller). „Serratur doppeit, drüsig; untere Blätter unterseits g an z drüsig, aber die Drüsen dünn, fein, bei obliterirendem Flaume rostbraun schimmernd. Zw oig e flaumig und sammt den Petiolen reich bestachelt. Daher wohl nur zu den Varietäten der $\boldsymbol{R}$. tomentosa Sm. (Keller, Rosen von Nied.Oest. p. 248 ff.) zu ziehen, wo sie eine zweite, der $R$. pseudocuspidata parallele Verbindungsform der var. floccida und anthracitica darstellt, also versus pseudocuspidatam (Crép.) $=R$. Seringeana Godr. Auch nach Gandoger's Tabulae rbod., wo sie auf $\boldsymbol{R}$. luteo-virens n. 4006 fällt, kann sie, abgesehen von dem ohnehin sehr allgemeinon Merkmale der flammigen Zweige, thatsächlich nur noch $R$. Seringeano Godr. sein, und ist mit $\mathbb{R}$. tomentosa f. scabriuscula Christ 
(non Baker) p. p. synonym. Mit der $R$. abietina Gren. hat diese Rose nur die krummen Stacheln gemein" (Keller).

Nächst dem Barakenspital bei Travnik (leg. Brandis).

$\gamma$. Rosa confusa Puget f. occupata Wiesb. Von der typischen $\boldsymbol{R}$. confusa, welche Déséglise (Cat. rais. n. 383) beschreibt, weicht unsere bosnische Pflanze in mehrfacher Beziehung ab. Die Mittelnerven der Blattunterseite sind fast stets drüsenlos, die Nebenblätter unterseits stark behaart (nicht "glabres"), mitunter auch mit Drüsen besetzt, auch die Deckblätter sind unterseits behaart. Die Kelchanhängsel sind nicht kürzer, sondern eher länger als die (nicht grosse, sondern kleine) Krone. Die Griffel sind dünn, behaart (nicht "glabres"). Die Blätter sind oben ziemlich dünn behaart, unten kurz- aber dicht weissfilzig.

Beim Barakenspital nächst Travnik an Hohlwegen (leg. Brandis). Ferner rom Artillerie-Lieutenant der Occupationsarmee, Grafen J. Platz auch auf dem Tarabovac entdeckt.

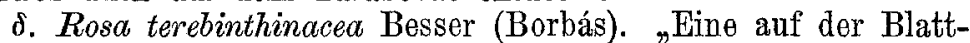
unterfläche armdrüsige oder meist ganz drüsenlose Modification" (Keller).

Am Tarabovac (leg. Brandis). Andere Exemplare vom selben Standorte wurden von H. v. Keller bezeichnet als "eine Form mit nur gespaltenen Blattzähnen und feblenden Drüsen auf der Unterseite dor meiston Blätter". - Als ich die Exemplare aus Bosnien erhielt, machten sie sogleich den Eindruck der völligen Uobereinstimmung mit einer 1879 bei Oedenburg von mir gefundensn and von Prof. v. Borbás als $R$. terebinthinacea bestimmten Pflanze (vide Borb. prim. p. 511). Ihre grossen Blumen sind zu auffallend und erinnern in der That, wie Borbás (l. c.) hervorhebt, an $R$. austriaca Crantz, sind aber viel heller rosenfarb als die der letzteren, welche auch viel niedriger ist. Rosa terebinthinacea wird zwar von Prof. Brandis anch als "niedriger Stranch" bezeichnet, wohl nur im Gegensatz zu den grossen Caninen etc.; der von mir bei Oedenburg beobachtete Strauch, der einzige in Ungarn bekannte, hatte jedoch eine Höhe von 1 bis 1.30 Met. - Die $R$. Genevensis Puget, welche von Borbás als grossblätterige, nur an den Nerven drüsige Varietät zur $\boldsymbol{R}$. terebinthinacea gestellt wird, wäre des letzteren Merkmales wegen mit unserer Rose fast übereinstimmend; nur ist die bosnische Pflanze vielmehr eine forma eglandulosa. Von Rapin wird die $\boldsymbol{R}$. Genevensis als $\boldsymbol{R}$. tomentosa $\times$ gallica gedeutet, was anf die bosnische Pflanze schon der Blüthezeit wegen gar nicht passt, da $R$. gallica, wenigstens unsere $R$. austriaca Crantz, die hier allein in Betracht kommen könnte, zu den spät- (erst nach den Rubiginosen) blühenden gehört. $R$. terebinthinacea hingegen beginnt, wenn nicht vor, so doch wenigstens gleichzeitig mit den Caninen, gehört also (mit der $R$. Zalana ${ }^{1}$ ) zu jenen Arten,

1) Vergl. Wiesb. "Zur Flora des Eisenburger Comitates" in Oe. b. Z. 1883 Nr. 2, S. 46 . f. 
welche in unseren Gegenden nach der $R$. pimpinellifolia am frühesten blühen. So wurde denn auch um Travnik die $\dot{R}$. austriaca zugleich mit $\boldsymbol{R}$. tomentosa f. occupata vom Grf. Platz erst den 7. Juni am Tarabovac und beide meist noch im Knospenzustande gefunden, während $R$. terebinthinacea ebendort von Prof. Brandis bereits am 27. Mai und zwar in voller Blüthenentfaltung gesammelt worden ist. Damit übereinstimmend ist auch die Rose von Oedenburg, welche am 5. Juni 1879 schon allgemein blühte, als die Caninen eben die ersten Blumen entfalteten. Bastarte aber halten nach den bisherigen Erfabrungen anch in der Blüthezeit die Mitte ein. Andererseits muss erwähnt werden, dass Exemplare, welche P. Brandis am 5. Juli am Tarabovac gesammelt hat, bereits vertrocknete und halbvertrocknete Fruchtstiele und Scheinfrüchte besitzen, ganz so, wie es an der $\boldsymbol{R}$. Kalksburgensis, der einzigen sicher hybriden Rose, die mir bekannt geworden, seit einer Reihe von Jahren beobachtet wird. Fortgesetzte Beobachtung wird somit hier besonders empfohlen.

E. R. farinulenta Crép.? - Fin sehr unvollständiges Zweiglein einer Tomentose, am 13. Juni blühend bei Travnik von P. Brandis gesammelt, das wohl von den vorhergehenden Varietäten verschieden ist, sich aber nicht näher bestimmen lässt, „ob es, wie es den Anschein hat, zu der bereits durch Sendtner aus Bosnien bokannten $R$. farinulenta gehört" (Keller).

15. Rosa floribunda (Steven ${ }^{1}$ ) Bess. enum. p. 68, n. 1525. Uober diesen höchst interessanten Fund einer in der ganzen Monarehie bisher unbekannten Rose wurde bereits in der Oesterr. botan. Zeitschr. 1882, S. 281 von mir und S. 376 von Keller vorläufig berjchtet. Damals stand nur eine brieflich zugesandte Probe zur Verfügung, die Herr Dix. Crépin für eine $\boldsymbol{R}$. tomentosa erklärte. Später langte vollständigeres Material an blühenden Zweigen an, wolches die zuerst ausgesprochene Ansicht Keller's nur bestätigte. Crépin, bei dem die drei Exemplare dieser Pflanze (Nr. 98-100) sich noch befinden, und der, was die übrigen eingesandten Proben betrifft, die Bestimmungen des Herin v. Keller sofort bestätigte, hat sich darüber noch nicht geäussert. - Zu einem Zweige, der sich unter den übrigen Pflanzen des P. Brandis befand, und dor gleichfalls dieselbe Mittelstellung zwischen den Tomentosen und Pubiginosen zeigte, wesshalb ich ihn ebenfalls als "R. micrantha?" an Herrn v. Keller sandte, bemerkt letzterer: "versus $R$. foribundam Besser. Abweichend von $\boldsymbol{R}$. tomentosa in den kahlen, freien Griffeln, starkem, noch im Herbar wahrnehmbaren Weingeruch ${ }^{2}$ ) bei oberseits schwach behaarten Blättern, in der schärferen, reichdrüsigen, seichten Serratur, den kleineren, kürzeren, kahleren Neben-

1) In Déséglise's Cat. rais. ist S. 291 Zeile 1 ein störender Druck-

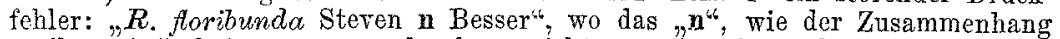
ergibt, "in "it heissen muss, durchaus nicht "non", wie in dergleichen Fällen gewöhnlich ist zu kürzen.

4) Weingeruch noch jetzt, Ostern 1883, wahrnehmbar.

W. 
blättern und schwächer filzigen Deckblättern, welch letztere kürzer als die Blüthenstiele sind; ferner in den sehr dünnen, feinen, weichen Drüsenhaaren und den flexuosen Zweigen. Aus diesen Gründen, und da auch die Besser'sche Diagnose vollkommen passt, als $R$. floribunda zu denten und zwar um so mehr, als auch aach Crépin (l. c. V. p. 264) die $R$. floribunda mit der $R$. tomentosa eine grosse Aehnlichkeit hat und nach Steven zahlreiche Eormen drüsiger Tomentosen in sich begreift, während sie nach Besser eine Mittelform der $\boldsymbol{R}$. rubiginosa und $\boldsymbol{R}$. tomentosa sein soll. Wir behalten uns diess betreffend vor, Crépin's endgiltige Bestimmung seiner Zeit mitzutheilen" (Koller).

Das eben beschriebene Exemplar wurde von einem Studenten, Ledić aus Grahovik dem P. Brandis (8. Juni) überbracht; die übrigen (noch in Brüssel befindlichen) sind wohl auch als bei "Travnik" (aber leider ohne nähere Angabe) und zwar als "häufig" vorkommend bezeichnet; sie seheinen jedoch nicht so häufig zu sein, da P. Brandis trotz wiederholten. Suchens keine Fruchtexemplare mehr finden konnte. Im Begleitschreiben zu den Blüthenexemplaren (vom 12. Juni) heisst es: "Weiche, glanzlose Blätter, mit (unten ziemlich dicht-, oben zerstrent-) behaarter Blattfläche wollig behaarten Blattstielen. Wächst strauchartig, etwa wie Berberis" (Brandis). - So weit ich mich erinnere, besitzen die bei Crép in befindlichen Exemplare grösseres und dunkleres Laub, stimmen aber im Uebrigen mit dem von Grahovik überein, so dass man sie nach Stachehn und Behaarung für eine Tomentose, im Uebrigen aber und namentlich in ihrer Tracht für eine Micranthe halten muss.

Hält man nun diese Thatsachen mit dem zusammen, was Besser (l. c.) unmittelbar an die Beschreibung der $R$. floribunda Stev, anfügt: "haberem pro varietate villosa $R$ osae rubiginosae, at aculei recti et petala ciliata contradicunt; caeterum fore Rosam villosam parvifoliam refert" - so ergibt sich, dass uns keine Rose bekannt ist, die mit der $\boldsymbol{R}$. floribunda Stev. näher verwandt wäre, als es die Pflanze von Travnik ist; sowie auch, dass das eben ron Keller beschriebene Exemplar der kleineren Blättchen wegen noch vollkommener mit der Pflanze Besser's ("parvifolia“) übereinstimmt, als jene, von denen in der Oe. b. Ztg. 1882 (1. c.) die Rede war, welch letztere man für eine Schattenform halten kann. Das bereits vorhandene Material einerseits, die Worte Besser's andererseits erlauben den Schluss auf das Vorhandensein einer Reibe von Varietäten (im modernen Sinne: einer Gruppe mit einer ganzen Formenreihe) dieser seltenen Art, welche zwischen Tomentosen und Rubiginosen einzuschalten ist. 